\title{
Enquête
}

Archives de la revue Enquête

4 | 1996

La ville des sciences sociales

\section{Le Centre civique de Bucarest}

De l'idée à la mémoire

The Civic Centre of Bucharest. From idea to memory

\section{Gérard Althabe}

\section{OpenEdition}

Journals

Édition électronique

URL : http://journals.openedition.org/enquete/823

DOI : 10.4000/enquete.823

ISSN : 1953-809X

Éditeur :

Cercom, Éditions Parenthèses

Édition imprimée

Date de publication : 1 novembre 1996

Pagination : 147-151

Référence électronique

Gérard Althabe, «Le Centre civique de Bucarest », Enquête [En ligne], 4 | 1996, mis en ligne le 12 juillet 2013, consulté le 02 mai 2019. URL : http://journals.openedition.org/enquete/823 ; DOI : 10.4000/ enquete.823

Ce document a été généré automatiquement le 2 mai 2019. 


\title{
Le Centre civique de Bucarest
}

\author{
De l'idée à la mémoire \\ The Civic Centre of Bucharest. From idea to memory
}

Gérard Althabe

1 Durant les années quatre-vingt, une grande partie du centre-ville de Bucarest a été démolie pour construire le Centre civique. En son cœur, sur une légère élévation, se dresse une bâtisse colossale, de forme parallépipédique et haute de 85 mètres : la Maison du Peuple, actuellement rebaptisée Maison du Parlement. Avec ses $330000 \mathrm{~m}^{2}$ et ses 900 salles, elle est en superficie le second bâtiment du monde; son volume, $2555000 \mathrm{~m}^{3}$, dépasse celui de la pyramide de Chéops... À partir de ce bâtiment, s'ouvre une avenue rectiligne, d'une dizaine de kilomètres, qui se perd dans l'horizon urbain. Plus large que les Champs-Élysées, elle est bordée d'immeubles de huit à dix étages et de bâtiments officiels. Autour de ce noyau, dans toutes les directions, c'est l'ensemble d'une ville de 2 millions d'habitants qui devait être recomposé.

2 L'opération a mobilisé des dizaines de milliers de travailleurs. L'ensemble des ressources du pays y ont été englouties, entraînant pénuries alimentaires, déshérence des infrastructures, paralysie des services publics, intervention accrue des services de sécurité politique. Les années quatre-vingt ont ainsi été, pour l'ensemble de la population roumaine, une période de terreur et de privations inouies. Durant l'hiver 1984-1985, le gaz permettant le chauffage urbain a ainsi été coupé, alors que la température est le plus souvent autour de 20 degrés au-dessous de zéro.

3 Le Centre civique - destruction d'un quartier central, expulsion et relogement de 40000 personnes - marque l'achèvement d'un mouvement de généralisation de la modernité urbaine. À Bucarest tout d'abord - avec les avenues perpendiculaires et circulaires élargies et bordées à l'infini d'immeubles, et la construction de grands ensembles à la périphérie sur l'emplacement de l'ancienne banlieue (le mahala) -, puis dans les villes de province, objet, à leur tour, d'opérations gigantesques, soit que leur centre ait été démoli et recomposé dans le sens de la modernité, soit qu'il ait été conservé et quelque peu abandonné, tandis qu'une ville entièrement nouvelle était construite à côté et l'ensemble urbain recomposé autour d'elle. Il faut articuler la construction du Centre civique au 
projet de systématisation des villages. Il s'agissait d'urbaniser la campagne. Les paysans voyaient leur maison détruite, et ils étaient regroupés dans de petites unités urbaines à proximité d'immeubles d'habitation et de bâtiments officiels. Ce projet a connu un début d'exécution dans les environs de Bucarest : Ceauscescu a présidé la première destruction d'une maison rurale en juin 1988.

Dans la perspective du régime qui était celle, imposée avec constance, d'une urbanisation relevant de l'architecture et de l'urbanisme fonctionnaliste, quel sens possédait donc la modernité urbaine? Replaçons-la dans le cadre du projet totalitaire, dont le régime se voulait le porteur et l'est resté jusqu'au bout, celui de la construction d'une société socialiste entièrement nouvelle passant par la destruction de la société existante. La modernité urbaine prend sens dans le cadre de ce projet. Elle véhicule des formes d'aménagement et des formes architecturales qui ne relèvent en rien des traditions. L'effacement de la mémoire est à l'œuvre dans ces formes, telle l'imposition d'un axe EstOuest à une ville se développant selon un axe Nord-Sud, qui est l'imposition d'une perspective spatiale cherchant à annuler celle qui existait auparavant. Il est possible d'évoquer à ce propos les constructivistes russes des années vingt, mais également la Charte d'Athènes, dans cette idée de rupture d'avec l'existant, de la constitution d'un monde social entièrement libéré des pesanteurs du passé, de la tradition et de l'histoire.

Il est nécessaire de conjuguer cette modernité urbaine au pouvoir qui l'initie et qui a fait de son engendrement l'un de ses objectifs : un espace urbain engendré par le pouvoir, ce qui signifie la destruction d'un autre espace engendré, lui, en quelque sorte de l'intérieur, par le développement du temps et le remodelage de la culture. Le pouvoir considère cette modernité urbaine comme le creuset de l'homogénéisation de la population. En elle, les différences se perdent comme se perd la singularité de chaque ville, comme se perd son identité. Nous ne savons plus si l'on est à Brasov ou à Braila, nous sommes exclus de la différence entre l'urbain et le rural, et, dans le phénomène de l'urbanisation des campagnes, se trouve éliminées la singularité et la diversité des villages. Et l'uniformité qui résulte de cela apparaît comme le miroir de l'homogénéité de la population.

Cette homogénéisation prend sens dans la relation au pouvoir. Elle constitue l'égalité de tous telle qu'elle émane de cette relation puis, dans le cadre de cette unification préalable engendrée par la relation au pouvoir, elle fait apparaître des différences, des entités collectives et individuelles. Ainsi joue le mécanisme de l'arrachement des hommes et des femmes à leur passé, à leurs traditions, à l'autonomie de leur regroupement, et qui se traduit par leur assujettissement au pouvoir et à la réorganisation de leur existence autour de cette nouvelle centralité.

Le pouvoir est le médiateur nécessaire de cette unification et il s'exerce dans une tension toujours plus grande vers la transcendance et la séparation : il est dans un autre monde. Le culte entourant le Président prend, dans les années quatre-vingt, les formes les plus extravagantes: sacralisation de sa parole, congrès du parti où tous les participants prennent ostensiblement des notes pendant que le Conducator pérore à la tribune, poèmes à sa gloire, tableaux où il n'est plus humain - jeune avec les jeunes, ouvrier avec les ouvriers, paysan avec les paysans, roi au panthéon des rois. La Maison du Peuple dans son gigantisme traduit cette tension unificatrice dans la construction telle que la modernité urbaine l'a constituée.

8 Il faut évaluer le sens de la conjonction du pouvoir et de la ville telle qu'elle se produit dans le Centre civique en considérant qu'elle représente une transformation structurelle de l'espace symbolique du pouvoir qu'est la ville. Le régime commence par affirmer sa 
domination en installant ses bâtiments et monuments dans l'espace de ceux de l'ancienne société comme la place où se trouve le Palais Royal et, face à lui, un peu décalé, le siège monumental du Comité central, ou le mausolée des héros du socialisme, au sommet d'une colline surmontée d'une colonne s'élevant verticalement, écrasant en quelque sorte la colline de la métropole où siège le centre de l'Église orthodoxe : le pouvoir se donnant à voir à travers la subordination du vaincu. Puis, dans le Centre civique, le pouvoir se représente lui-même dans une solitude, un soliloque inquiétant. Inscrit dans la symbolique, s'opère le passage d'une situation dans laquelle le pouvoir s'exerce sur une société qui contient le passé et une acceptation de son altérité, à une autre dans laquelle le pouvoir a recréé la société où il s'exerce et résorbe l'altérité.

9 Dans le cadre de la dialectique entre le pouvoir tendant à son propre isolement et une population prise dans le processus d'homogénéisation, trouve place une couche dominante, la nomenklatura, qui, protégée, vit dans des villas et des aires urbaines gardées. Elle bénéficie d'une ségrégation spatiale et sociale. C'est une minorité aspirée par le pôle du pouvoir et, par là même, elle est orientée de plus en plus vers son centre, le Président, le couple présidentiel, sa famille. Elle s'établit dans leur proximité où elle est soumise aux fluctuations politiques, à la rotation des postes que favorise l'anonymat relatif de la scène publique, occupée seulement par le couple souverain.

Nous ne pouvons manquer de souligner la contradiction entre ce projet totalitaire, axé sur l'effacement de la société existante, de ses racines, de leur mémoire, et l'effort que le régime déploie, surtout depuis les années soixante-dix, pour s'enraciner dans la société et pour surmonter la violence fondatrice qui se perpétue: l'occupation soviétique, la brutalité des années cinquante avec ses camps de concentration et ses exécutions, la brutalité de la collectivisation des terres. Cet enracinement culturel est recherché par l'invocation du village et du passé. L'histoire nationale est réhabilitée et, détaché de l'histoire du parti et de ses figures, Ceauscescu s'installe dans la continuité des souverains. Ces pratiques symboliques atteignent leur apogée dans ces mêmes années quatre-vingt qui voient la construction du Centre civique : ainsi exalte-t-on le village et sa production artistique dans le même temps qu'on les voue à la disparition par l'urbanisation violente des campagnes. Un signe de cette contradiction : la destruction du monastère historique de Vacaresti alors que sa restauration minutieuse a duré sept années et vient de s'achever. Le sort réservé aux églises orthodoxes se trouvant dans l'axe de construction du Centre civique en témoigne également : les unes sont enclavées dans les immeubles, d'autres sont détruites, comme celle de la Sainte Vendredi, d'autres enfin sont déplacées comme cette église Mihai Voda dont le déplacement sur près de 300 mètres a donné des images saisissantes. Cette relative réticence à démolir les églises a deux raisons: d'une part la poursuite contradictoire de légitimation historique l'orthodoxie est une composante de la culture nationale, même si cette composante n'est pas officiellement acceptée -, d'autre part le signe de la résistance de la société à l'entreprise même de destruction dont elle est l'objet.

11 J'ai tenté une interprétation de ce lieu urbain et je suis resté dans la perspective du pouvoir de l'époque et de sa contradiction. Je n'ai fait là qu'œuvre d'archéologue. Le régime responsable de la construction du Centre civique a disparu et le sens que je déchiffre renvoie à un projet de société qui a lui-même disparu, le Centre civique étant devenu une sorte de lieu de mémoire. Ce n'est donc là qu'une approche partielle qui reste dans la perspective du pouvoir constructeur: d'autres directions d'analyses sont nécessaires. Il faudrait sortir de cette perspective et confronter cette réalisation non plus 
au sens qu'elle a pour ses constructeurs mais au contexte - la ville et le village - dans lequel se placent cette modernité urbaine et le rêve d'homogénéisation qui s'y inscrit. Enfin qu'en est-il maintenant de ce Centre civique, des immeubles, des avenues et du palais? Comment ceux qui les ont habités ont-ils réinvesti et donné sens aux immeubles de la modernité urbaine ? Comment le sens que leur donnaient les dominants a-t-il été subverti et décomposé?

\section{RÉSUMÉS}

La destruction et la reconstruction du centre-ville de Bucarest durant les années quatre-vingt sont un exemple du lien entre le despotisme, l'urbanisme et l'architecture. Alors que s'accumulent, à Varsovie et à Moscou en particulier, les signes du crépuscule des régimes communistes d'Europe, cette tentative folle d'assurer une pérennité de plus en plus aléatoire montre comment un urbanisme qui, à travers ses formes, rompt avec le passé est une des modalités de la mise en œuvre du projet totalitaire dont le régime caucescite était le porteur. Il doit alors affronter une contradiction fondamentale entre un projet totalitaire visant à la création d'une société nouvelle, libérée du passé et un pouvoir contraint de forger sa légitimité dans un nationalisme qui l'amène à manifester sa continuité avec le passé et son enracinement dans une culture roumaine exclusivement rurale.

The destruction and reconstruction of the city centre during the 1980's is an example of the link between despotism, urbanisation and architecture. As signs of the twilight of Europe's communist regimes gather, particularly in Warsaw and Moscow, the crazy attempt to ensure an increasingly uncertain perpetuity demonstrates how a town planning which breaks with the past in its forms is one of the ways of the application of a totalitarian project led by Ceaucescu's regime. The latter then has to affront a fundamental contradiction between a totalitarian project aiming at the creation of a new society liberated from the past and a power forced to forge its legitimacy in a nationalism. This leads the regime to manifest its continuity with the past and its roots in an exclusively rural roumanian culture.

\section{AUTEUR}

\section{GÉRARD ALTHABE}

Gérard Althabe (EHESS) a ouvert depuis 1992 un chantier de recherche en Roumanie. Dans les années soixante et soixante-dix, il a travaillé sur l'anthropologie sociale en Afrique centrale et à Madagascar puis, à la fin des années soixante-dix, il s'est consacré à l'urbain et à l'entreprise. Ses principaux ouvrages sont Oppression et libération dans l'imaginaire : les communautés villageoises de la côte orientale de Madagascar [1969], Paris, F. Maspero, 1982 ; avec B. Légé et M. Selim, Urbanisme et réhabilitation symbolique : Ivry, Boulogne, Amiens [1984], Paris, L'Harmattan, 1993 ; avec J.-L. Comolli, Regards sur la ville, Paris, Éditions du Centre Georges Pompidou, 1994. 